
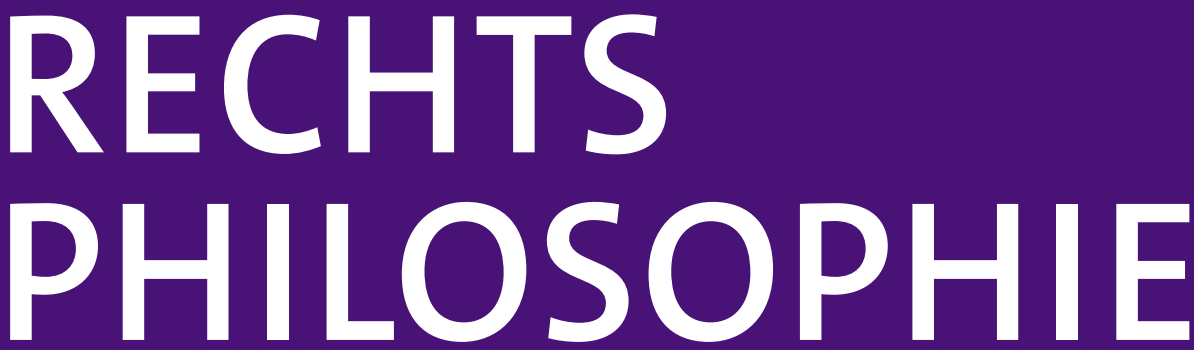

\title{
Zeitschrift für die Grundlagen des Rechts
}

Herausgegeben von

Alexander Aichele

Martin Borowski

Elisabeth Holzleithner

Joachim Renzikowski

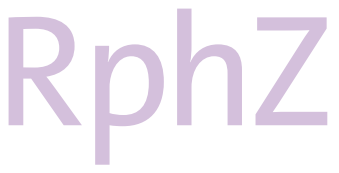

\section{$3 \mid 2019$}

5. Jahrgang | Seite 247-351

ISSN 2364-1355
Thema:

Autonomie

Bernhard Opolony

Gesellschaft und Vergessen. Überlegungen zu

Selbstbestimmung und Demenz im Menschenbild des

Grundgesetzes

Tobias Herbst

Autonomie und broad consent in der medizinischen Forschung

Moritz Heepe

Strafrecht als Heilkunst. Zur platonischen Strafgerechtigkeit

Manuel M. Güntert

Das Jenseits des Rechts

Stefan Brunnhuber

Böckenförde 2.0: Offene Gesellschaften und autokratische

Experimente

\section{Rezensionen}

Tatjana Hörnle:

Boris Burghardt, Zufall und Kontrolle, 2018

Katja Stoppenbrink:

Franziska Dübgen, Theorien der Strafe zur Einführung, 2016

Claus Dierksmeier:

Nikolaus Knoepffler, Würde und Freiheit, 2018 


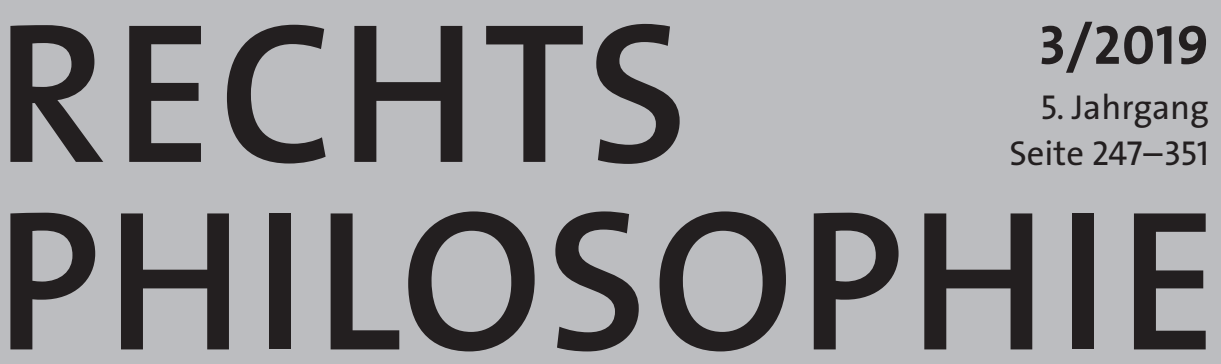

\section{Zeitschrift für die Grundlagen des Rechts}

Herausgegeben von PD Dr. Alexander Aichele, Martin-Luther-Universität Halle-Wittenberg| Prof. Dr. Martin Borowski, Universität Heidelberg | Univ.-Prof. Dr. Elisabeth Holzleithner, Universität Wien | Prof. Dr. Joachim Renzikowski, Martin-Luther-Universität Halle-Wittenberg unter Mitarbeit von Prof. Dr. Wolfgang Ertl, Keio University Tokyo | Prof. Dr. Jean François Kervégan, Université Panthéon-Sorbonne (Paris 1) | Prof. Dr. Christian Krijnen, Vrije Universiteit Amsterdam | Dr. Anne Kühler, Universität Zürich | Prof. Dr. Joachim Lege, Universität Greifswald | Prof. Dr. Georg Mohr, Universität Bremen | Prof. Dr. Stanley L. Paulson, Universität Kiel | Prof. Dr. Beate Rössler, Universiteit van Amsterdam | Prof. Dr. Kurt Seelmann, Universität Basel

Schriftleitung: Prof. Dr. Joachim Renzikowski (V.i.S.d.P.), Martin-Luther-Universität HalleWittenberg, Lehrstuhl für Strafrecht, Rechtsphilosophie/Rechtstheorie, Universitätsplatz 6, D-06108 Halle (Saale), Tel. 0345/5523130, E-Mail: Joachim.Renzikowski@jura.uni-halle.de

\section{Inhaltsverzeichnis}

\section{Thema: Autonomie}

Bernhard Opolony:

Gesellschaft und Vergessen. Überlegungen zu Selbstbestimmung und Demenz im Menschenbild des Grundgesetzes. . . . . . . . . . . . . . . . . . . . . 251

\section{Tobias Herbst:}

Autonomie und broad consent in der medizinischen Forschung. . . . . . . . . 271

Moritz Heepe:

Strafrecht als Heilkunst. Zur platonischen Strafgerechtigkeit . . . . . . . . . . 288

\section{Manuel M. Güntert:}

Das Jenseits des Rechts . . . . . . . . . . . . . . . . . . . . . . . . . . 308

Stefan Brunnhuber:

Böckenförde 2.0: Offene Gesellschaften und autokratische Experimente . . . . . 326

\section{Rezensionen:}

Tatjana Hörnle:

Boris Burghardt, Zufall und Kontrolle, 2018. . . . . . . . . . . . . . . . . . . 333 
Katja Stoppenbrink:

Franziska Dübgen, Theorien der Strafe zur Einführung, 2016 . . . . . . . . . . . . . 340

Claus Dierksmeier:

Nikolaus Knoepffler, Würde und Freiheit, 2018

\section{Impressum RphZ - Zeitschrift für die Grundlagen des Rechts}

Herausgegeben von: PD Dr. Alexander Aichele, Martin-Luther-Universität Halle-Wittenberg | Prof. Dr. Martin Borowski, Universität Heidelberg | Univ.-Prof. Dr. Elisabeth Holzleithner, Universität Wien | Prof. Dr. Joachim Renzikowski, Martin-LutherUniversität Halle-Wittenberg

unter Mitarbeit von Prof. Dr. Wolfgang Ertl, Keio University Tokyo | Prof. Dr. Jean François Kervégan, Université PanthéonSorbonne (Paris 1) | Prof. Dr. Christian Krijnen, Vrije Universiteit Amsterdam | Dr. Anne Kühler, Universität Zürich | Prof. Dr. Joachim Lege, Universität Greifswald | Prof. Dr. Georg Mohr, Universität Bremen | Prof. Dr. Stanley L. Paulson, Universität Kiel | Prof. Dr. Beate Rössler, Universiteit van Amsterdam | Prof. Dr. Kurt Seelmann, Universität Basel

Schriftleitung: Prof. Dr. Joachim Renzikowski (V.i.S.d.P.), Martin-Luther-Universität Halle-Wittenberg, Lehrstuhl für Strafrecht, Rechtsphilosophie/Rechtstheorie, Universitätsplatz 6, D-06108 Halle (Saale), Tel.0345/5523130, E-Mail: Joachim.Renzikowski@ jura.uni-halle.de

www.rphz.de

Erscheinungsweise: Vierteljährlich

Bezugspreise 2019: Jahresabonnement incl. Onlinezugang für Privatbezieher 155,- $€$; für Institutionen 219,- $€$; für Unternehmen Preis auf Anfrage; Einzelheft 41,- $€$. Alle Preise verstehen sich incl. MwSt., zzgl. Vertriebskostenanteil 12,- $€$, plus Direktbeorderungsgebühr Inland 1,65 € p.a. Beihefte, die zu diesem Titel erscheinen, werden den Abonnenten und Abonnentinnen mit einem Vorzugspreis automatisch zugesandt und können bei Nichtgefallen zurückgegeben werden.

Bestellmöglichkeit: Bestellungen beim örtlichen Buchhandel oder direkt bei der Nomos Verlagsgesellschaft Baden-Baden

Kündigungsfrist: jeweils drei Monate vor Kalenderjahresende

Bankverbindung generell: Zahlungen jeweils im Voraus an Nomos Verlagsgesellschaft, Postbank Karlsruhe: IBAN DE07 66010075 0073636751 (BIC PBNKDEFF) oder Sparkasse Baden-Baden Gaggenau: IBAN DE05 662500300005002266 (BIC SOLADES1BAD). Druck und Verlag: Nomos Verlagsgesellschaft mbH \& Co. KG, Waldseestr. 3-5, D-76530 Baden-Baden, Telefon (07221) 2104-0/ Fax (07221) 2104-27, E-Mail: nomos@nomos.de

Anzeigen: Sales friendly Verlagsdienstleistungen, Pfaffenweg 15, 53227 Bonn, Telefon (0228) 978980/Fax (0228) 9789820, EMail: roos@sales-friendly.de.

Urheber- und Verlagsrechte: Die Zeitschrift sowie alle in ihr enthaltenen einzelnen Beiträge und Abbildungen sind urheberrechtlich geschützt. Jede Verwertung, die nicht ausdrücklich vom Urheberrechtsgesetz zugelassen ist, bedarf der vorherigen Zustimmung des Verlags. Mit der Annahme zur Veröffentlichung überträgt der Autor/die Autorin dem Verlag das ausschließliche Verlagsrecht für die Zeit bis zum Ablauf des Urheberrechts. Eingeschlossen sind insbesondere auch das Recht zur Herstellung elektronischer Versionen und zur Einspeicherung in Datenbanken sowie das Recht zu deren Vervielfältigung und Verbreitung online oder offline ohne zusätzliche Vergütung. Nach Ablauf eines Jahres kann der Autor/die Autorin anderen Verlagen eine einfache Abdruckgenehmigung erteilen; das Recht an der elektronischen Version verbleibt beim Verlag. Vgl. dazu auch die Angaben unter www.nomos.de/urheberrecht.

Namentlich gekennzeichnete Beiträge geben nicht in jedem Fall die Meinung der Herausgeber/Redaktion oder des Verlages wieder.

Unverlangt eingesendete Manuskripte - für die keine Haftung übernommen wird - gelten als Veröffentlichungsvorschlag zu den Bedingungen des Verlages. Die Redaktion behält sich eine längere Prüfungsfrist vor. Eine Haftung bei Beschädigung oder Verlust wird nicht übernommen. Bei unverlangt zugesandten Rezensionsstücken keine Garantie für Besprechung oder Rückgabe. Es werden nur unveröffentlichte Originalarbeiten angenommen.

Die Verfasserinnen und Verfasser erklären sich mit einer nicht sinnentstellenden redaktionellen Bearbeitung einverstanden. Der Nomos Verlag beachtet die Regeln des Börsenvereins des Deutschen Buchhandels e.V. zur Verwendung von Buchrezensionen. 


\section{RphZ - Rechtsphilosophie}

\section{Zeitschrift für die Grundlagen des Rechts}

\section{Editorial}

Autonomie ist ein zentraler Begriff des Rechts. Über seinen Inhalt herrscht allerdings keine Einigkeit. Genauer gesagt dürfte es viele verschiedene Begriffe von Autonomie geben, von der schlichten Freiheit, aus verschiedenen Verhaltensalternativen zu wählen, bis hin zum Vermögen, sich zu sittlichem Handeln zu bestimmen. Autonomie drängte sich also als Oberthema auf.

Leider wird die Autonomie nur in zwei Facetten behandelt. Bernhard Opolony erörtert die höchst aktuelle Frage, ob und inwieweit dementen Personen ein selbstbestimmtes Leben ermöglicht werden kann. Zwar ist die Selbstbestimmung ein wesentliches Element des Menschenbildes des Grundgesetzes. Aber die Betonung intellektueller und rationaler Fähigkeiten sei, so Opolony, zu eng. Vielmehr gehe es um Selbstaktualisierung und damit müsse man den motivationalen Grundlagen mehr Aufmerksamkeit schenken als bisher. Tobias Herbst befasst sich mit der Einwilligung in medizinische Versuche. Üblicherweise wird hier ein ,informed consent“ verlangt. Aber was gilt für die Einwilligung in künftige Studien mit Körpermaterialproben und Daten eines Probanden, deren Verwendungsmöglichkeiten und Erkenntnisse zum Zeitpunkt des körperlichen Eingriffs noch nicht absehbar sind? Herbst plädiert für die Anerkennung eines „broad consent“, soweit die Probanden über die Ungewissheit aufgeklärt und sie bewusst in Kauf genommen haben.

Der Beitragsteil beginnt mit einer Studie über das Verständnis von Strafe und Strafrecht bei Platon, der mit dem Oberthema in einem gewissen Zusammenhang steht. Moritz Heepe zeigt nämlich, dass Platon zufolge ein Straftäter nicht selbstbestimmt handeln kann, weil er damit auch seiner eigenen Wohlfahrt zuwiderhandelt. Sofern das Verbrechen nicht aus Unwissenheit oder aus Zwang begangen wird, muss der Verbrecher verrückt sein. Damit ist er therapiebedürftig. Das Verständnis von Strafe als einer Art Therapie enthält jedoch einige unerfreuliche Implikationen. Manuel M. Güntert diskutiert das „Jenseits des Rechts“. Ihm zufolge gründet sich das Recht in jenen bösen Gedanken, deren Ausführung es verbietet. Daher könne es auch nur aus diesen bösen Gedanken heraus erkannt werden. Umgekehrt würden diese Gedanken erst durch das Recht für böse erklärt. Die Beiträge schließen mit einem nachdrücklichen Plädoyer für die Offene Gesellschaft von Stefan Brunnhuber. In Anlehnung an ein Zitat von Böckenförde erklärt er, dass auch Autokratien von Voraussetzungen leben, die sie nicht selbst bereitstellen können, nämlich von den Errungenschaften einer freien Presse, freier Wissenschaft, des öffentlichen Diskurses, freier Märkte, Kultur und Bildung, kurz: den Leistungen der Offenen Gesellschaft.

Im Rezensionsteil stellt Tatjana Hörnle die Habilitationsschrift von Boris Burghardt über „Zufall und Kontrolle“ vor. Katja Stoppenbrink empfiehlt die Studie von Franziska Dübgen über Straftheorien zur Lektüre. Schließlich bespricht Claus Dierksmeier die Studie von Nikolaus Knoepffler über „Würde und Freiheit“". 
Das Oberthema des letzten Hefts in diesem Jahr ist der „Rechtsfall“ mit so unterschiedlichen Aspekten wie der praktischen Arbeit am Fall oder dem Zusammenhang von Norminterpretation und Lebenssachverhalt, für den Engisch die berühmte Formulierung vom „Hin und Herwandern des Blickes“ geprägt hat. Da die RphZ immer auch Beiträge ,außer der Reihe“ enthält, sind Sie herzlich eingeladen, sich zu beteiligen. Texte reichen Sie bitte möglichst zahlreich in elektronischer Form bei renzikowski@jura.uni-halle.de ein. Auf der Homepage renzikowski.jura.uni-halle.de finden Sie einen Link zu den Hinweisen für die Autoren, deren Beachtung die redaktionelle Arbeit erleichtert. Wer sich ein Bild von der RphZ machen möchte, klickt auf:

https://www.rphz.nomos.de.

Halle/Heidelberg/Wien, September 2019

\author{
Alexander Aichele, \\ Martin Borowski, \\ Elisabeth Holzleithner, \\ Joachim Renzikowski
}

